\title{
Antimalarial Flavonoid-Glycoside from Acacia pennata with Inhibitory Potential Against PfDHFR-TS: An In- silico Study
}

\author{
James H. Zothantluanga ${ }^{1, *(\mathbb{D})}$, S Keerthic Aswin ${ }^{2 \mathbb{D}}$, Mithun Rudrapal ${ }^{3, * \mathbb{C}}$, Dipak Cheita ${ }^{1(\mathbb{C}}$ \\ 1 Department of Pharmaceutical Sciences, Faculty of Science and Engineering, Dibrugarh University, Dibrugarh 786004, \\ Assam, India \\ 2 Council of Scientific and Industrial Research (CSIR)-Institute of Genomics and Integrative Biology, New Delhi 110020, \\ India \\ 3 Department of Pharmaceutical Chemistry, Rasiklal M. Dhariwal Institute of Pharmaceutical Education \& Research, Pune \\ 411019, Maharashtra, India \\ * Correspondence: jameshztta@gmail.com (J.H.Z.); rsmrpal@gmail.com (M.R.)
}

Scopus Author ID 36524068400

Received: 9.08.2021; Revised: 15.09.2021; Accepted: 20.09.2021; Published: 16.10.2021

\begin{abstract}
Drug resistance, toxicity, and adverse effects of current antimalarial drugs have mandated the need to search for newer antimalarial agents. The present study aims to identify promising flavonoid-glycosides (FGs) from Acacia pennata as possible antimalarial agents effective against PfDHFR-TS (PDB ID: 3DGA) by in-silico studies. The co-crystal inhibitor (RJ1) of PfDHFR-TS was used as the reference standard compound. A compound library of 17 FGs reported to be isolated from A. pennata was prepared and subjected to molecular docking simulation studies. PyRx 0.8 and AutoDock Vina revealed Pinocembrin-7-O- $\beta$-D-glucopyranoside (FG17) as the best PfDHFR-TS inhibitor with a binding affinity of $-10.4 \mathrm{kcal} / \mathrm{mol}$ and $-10.8 \mathrm{kcal} / \mathrm{mol}$, respectively. In both methods, FG17 has a better binding affinity than the co-crystal inhibitor, RJ1 $(-7.9 \mathrm{kcal} / \mathrm{mol})$. The docking protocols were validated by RMSD calculation with Discovery Studio Visualizer software (2020). FG17 interacted with amino acids ALA16, LEU40, SER167, GLY41, GLY44, MET55, PHE58, ILE112, LEU119, GLY166, and TYR170 at the active binding site of PfDHFR-TS. Further, FG17 was computed as a non-toxic, bioavailable, synthetically accessible compound and a better enzyme inhibitor than RJ1. Hence, we conclude that FG17 may be used as a lead scaffold to design antimalarial agents against PfDHFR-TS in the future.
\end{abstract}

Keywords: antimalarial; Acacia pennata; flavonoid-glycoside; PfDHFR-TS; in-silico.

(C) 2021 by the authors. This article is an open-access article distributed under the terms and conditions of the Creative Commons Attribution (CC BY) license (https://creativecommons.org/licenses/by/4.0/).

\section{Introduction}

Malaria burdens the healthcare system with approximately 229 million cases and 409,000 deaths in 2019 [1]. Malaria is caused by different protozoan parasites, among which Plasmodium falciparum and Plasmodium vivax account for the most number of cases, serious illnesses, and deaths [2]. Although both species of Plasmodium can cause a severe form of malaria [3, 4], $P$. falciparum can lead to organ damage, and in the case of a pregnant woman, fetal death may even occur [5].

Although there are drugs available to treat malaria, Plasmodium parasites had developed drug resistance against pyrimethamine, proguanil, chloroquine, mefloquine, atovaquone [6], quinine, and artemisinin [7]. In addition to drug resistance, other factors such https://biointerfaceresearch.com/ 
as toxicity, adverse effects, and other problems further extend the drawback of the present antimalarial drugs [8-11]. As several studies continue to report antimalarial drug resistance, the present antimalarial drugs seem to lose their efficacy against emerging drug-resistant parasites [12-14]. The World Health Organization (WHO) had also addressed antimalarial drug resistance as a serious problem [15].

Due to various problems associated with the present antimalarial drugs, natural productbased drug discovery has once again received a renewed interest from scientists with the hope of discovering new antimalarial leads [16]. Two of the most widely and successfully used antimalarial drugs, viz. quinine, and artemisinin were obtained from traditional medicinal plants [17, 18]. Hence, researchers have adopted multiple approaches to identify new antimalarial leads from medicinal plants $[19,20]$. In-silico techniques such as high throughput screening, molecular docking, toxicity analysis, bioavailability estimation, and bioactivity prediction have played an important role in identifying and developing antimalarial leads [21, 22].

Dihydrofolate reductase-thymidylate synthase (DHFR-TS) is a bifunctional enzyme essential for folate biosynthesis in P. falciparum [23]. It is well known that the inhibition can treat malaria of DHFR [24]. Therefore, DHFR-TS of $P$. falciparum (Pf) represents a promising drug target to identify new antimalarial leads [25]. With the aid of in-silico techniques, a library of prepared compounds may be virtually screened against PfDHFR-TS to identify a potential antimalarial lead. In-silico techniques provide valuable insights into the pharmacological profile of plant bioactive compounds [26].

Acacia pennata (L.) willd is a medicinal plant that is traditionally used to treat malaria [27]. However, the medicinal plant is still not investigated for its antimalarial activity. Several studies had reported that many flavonoid-glycosides (FGs) were isolated from A. pennata [28-30]. FGs reported to be isolated from A. pennata includes apigenin 6-C-[2"-O-(E)feruloyl- $\beta$-D-glucopyranosyl]-8- $C$ - $\beta$-glucopyranoside $\quad$ (FG1), isorhamnetin $3-O-\alpha$-Lrhamnopyranoside (FG2), kaempferol 3-O- $\alpha$-L-rhamnopyranosyl-( $1 \rightarrow 4)-\beta$-Dglucopyranoside (FG3), isovitexin (FG4) [28], quercetin 3-O- $\beta$-D-glucopyranosyl-4- $O$ - $\beta$-Dglucopyranoside (FG5) [30], koaburanin (FG6), 5,7-dihydroxyflavone 7-O- $\beta$-Dglucopyranosyl-8-C- $\beta$-boivinopyranoside (FG7), 5,7-dihydroxyflavone $\quad 6-\mathrm{C}-\beta$ boivinopyranosyl-7-O- $\beta$-D-glucopyranoside (FG8), (2R)-4',7-dihydroxyflavan- $(4 \mathrm{a} \rightarrow 8)$ (2R,3S)-3,5,7-trihdyroxyflavan-3"- $O$ - $\alpha$-L-rhamnopyranoside $\quad$ (FG9), (2S)-5,7dihydroxyflavan-7-O- $\beta$-D-glucopyranoside-(4a $\rightarrow 8)$-epiafzelechin-3-O-gallate (FG10), (2R, $3 \mathrm{~S}$ )-3,5,7-trihdyroxyflavan-3- $O$ - $\alpha$-L-rhamnopyranoside (FG11), quercetin 4'-O- $\alpha$-Lrhamnopyranosyl-3-O- $\beta$-D-allopyranoside (FG12), quercetin-3-O- $\beta$-D-glucopyranoside (FG13), quercetin-3- $O$ - $\alpha$-L-rhamnopyranoside (FG14), chrysin-7- $O$ - $\beta$-D-glucopyranoside (FG15), kaempferol 3-O- $\alpha$-L-rhamnopyranoside (FG16) and pinocembrin-7-O- $\beta$-Dglucopyranoside (FG17) [29].

The chemical structure of all the FGs is given in Figure 1. Flavonoid-glycosides are among the different classes of secondary plant metabolites whose antimalarial activities have been reported by several studies [31-34]. Lupinifolinol, a flavonoid isolated from Mundulea sericea was reported to inhibit chloroquine-sensitive $(6.6 \mu \mathrm{M})$, and resistant $(2.0 \mu \mathrm{M})$ strains of $P$. falciparum in an in-vitro assay [31]. Quercitrin and myricitrin also showed in-vitro antimalarial activity against $P$. falciparum [32]. The present study aims to identify flavonoidglycoside (FG) reported from $A$. pennata as a possible antimalarial agent against $P f$ DHFR-TS by in-silico investigations. 


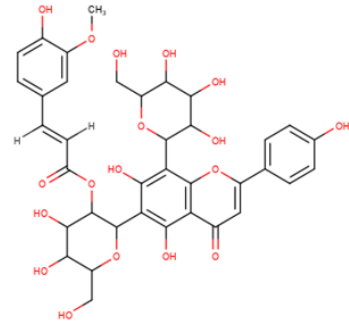

FG1

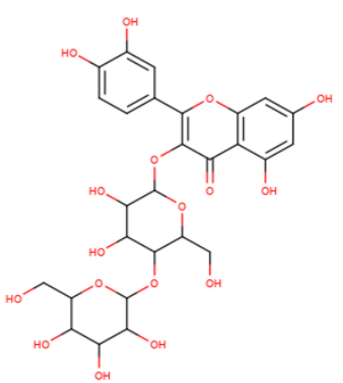

FG5

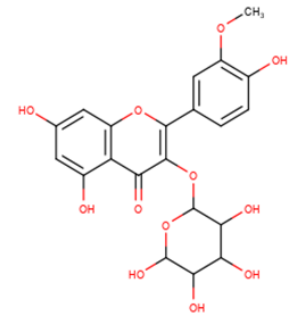

FG2

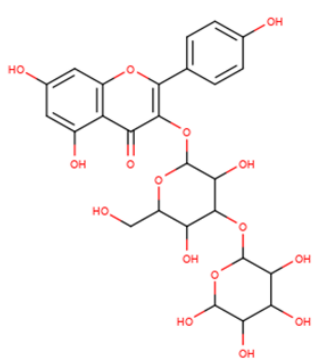

FG3

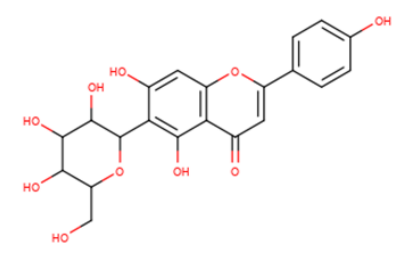

FG4

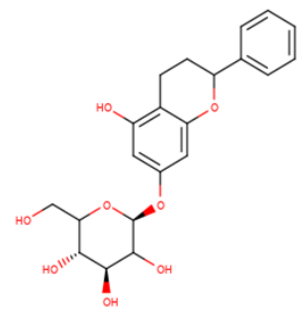

FG6

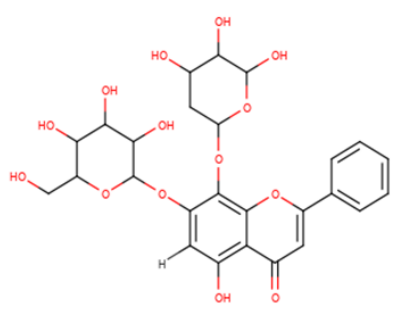

FG7

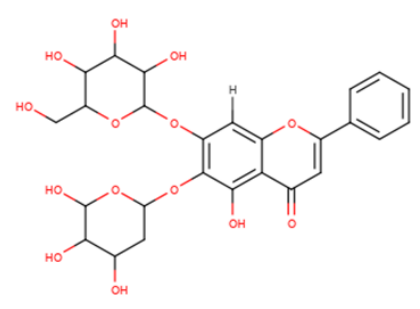

FG8

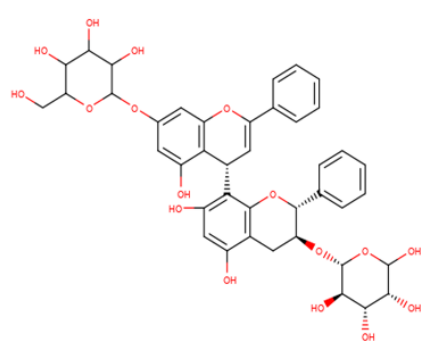

FG9

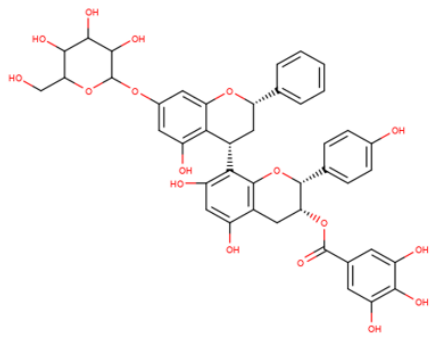

FG10

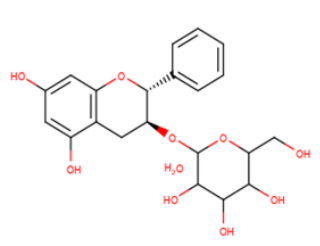

FG11

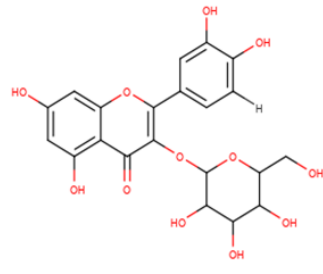

FG13

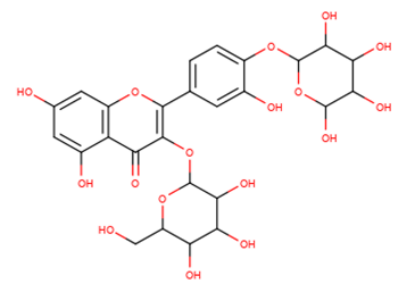

FG12

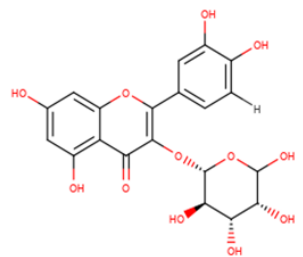

FG14

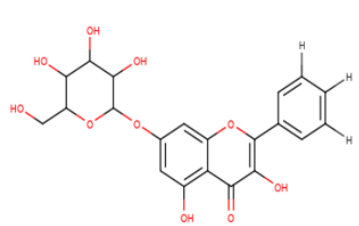

FG15

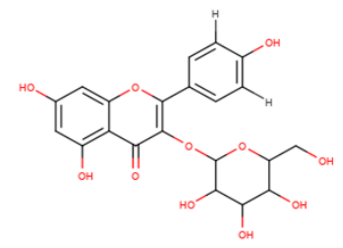

FG16<smiles>O=C1C[C@@H](c2ccccc2)Oc2cc(O[C@@H]3OC(CO)C(O)C(O)C3O)cc(Br)c21</smiles>

FG17

Figure 1. Compound library of FGs reported being isolated from A. pennata.

\section{Materials and Methods}

\subsection{Retrieval of the target protein.}

The crystal structure of PfDHFR-TS bearing a PDB ID: 3DGA was retrieved from the Research Collaboratory for Structural Bioinformatics-Protein Data Bank (RCSB-PDB) database [35]. The target protein is made up of four chains, i.e., chain A, chain B, chain $C$, and chain $\mathrm{D}$. The target protein is complexed with a co-crystallized inhibitor, i.e., RJ1. RJ1 has an 
experimental $\mathrm{IC}_{50}$ value of $29.9 \mu \mathrm{M}$ [35]. RJ1 was used as a positive control throughout the entire study. The protein was downloaded in '.pdb' format.

\subsection{Pre-processing of protein.}

Pre-processing of the target protein was done with the Discovery Studio Visualizer software [36]. Chain B, chain C, and chain D were deleted from the target protein. For the study, only chain A of PfDHFR-TS was used. From the target protein, water and heteroatoms were removed. This was followed by the addition of hydrogens. At this stage, the coordinates of the active binding site of the target protein were also identified with the Discovery Studio Visualizer software. The active site coordinates $(\mathrm{x}=27.54097 ; \mathrm{y}=4.46727 ; \mathrm{z}=59.37166)$ with little adjustments was noted down in notepad and was saved as '.txt' document for future use. The target protein was saved in '.pdb' format.

\subsection{Preparation of compound library.}

A compound library of the 17 FGs and RJ1 used in the study was prepared manually with MarvinSketch 20.10 software [37]. After each structure was drawn, the correctness of all the chemical structures was evaluated with the add-on 'Structure checker' available on the MarvinSketch software. The SMILES ID was also saved for future use. The prepared FGs were saved in 'MDL SDfile *.sdf *.sd' format.

\subsection{Molecular docking simulation studies.}

For the present study, molecular docking simulation studies were carried out with the algorithms of AutoDock Vina by two different methods using two independent software as depicted below.

\subsubsection{Docking on PyRx 0.8.}

PyRx 0.8 software is a virtual screening tool that uses AutoDock Vina to conduct molecular docking simulation studies [38, 39]. The energy of all the ligands was minimized using the default parameters (Force field = Universal Force Field; Optimization algorithm = Conjugate; Total number of steps $=200$; Number of steps for update $=1$; Stop is energy difference is less than $=0.1$ ) of the PyRx 0.8 software. After the energy minimization process was completed, the ligands were converted into the '.pdbqt' file format.

The pre-processed protein was loaded onto the 3D scene of the virtual screening platform of the software. The target protein was converted into the '.pdbqt' file format by making the target protein the macromolecule. In the Vina search space of the PyRx 0.8 software, the pre-defined active binding site coordinates were used to adjust the 3D affinity grid box to cover the entire active site residues. The size of the 3D affinity grid box was kept default at $25 \AA$. The exhaustiveness was also kept default at 8. Finally, the molecular docking simulation was carried out as the standard protocols described for the PyRx 0.8 software [40].

\subsubsection{Docking on AutoDock Vina.}

AutoDockTools1.5.6 offers a simple graphical user interface to prepare proteins and ligands for molecular docking simulation studies [41]. Protein preparation was done using the protein preparation wizard tool in Schrodinger Maestro [42]. The target protein was prepared 
for docking by minimizing its energy with Schrodinger Maestro (Forcefield= OPLS_2005 and the minimization proceeds until there is no significant change). Kollman charges were added to the target protein with AutoDockTools1.5.6, then converted to 'pdbqt' file format.

The energy of all the ligands was minimized using the default parameters (Force field $=$ Universal Force Field; Optimization algorithm = Conjugate; Total number of steps $=200$; Number of steps for update $=1$; Stop is energy difference is less than $=0.1$ ) of the PyRx 0.8 software. To all the ligands, hydrogens and Gasteiger charges were added with AutoDockTools1.5.6. Following this, the ligands were converted to the '.pdbqt' file format.

The size of the grid box was set to 18,14 , and 18 points in $\mathrm{X}, \mathrm{Y}$, and $\mathrm{Z}$ dimensions, respectively. The active binding site coordinates previously defined with Discovery Studio Visualizer software were used. Finally, AutoDock Vina stand-alone package was used to carry out the molecular docking simulation studies [38].

\subsection{Validation of docking protocol.}

As described by several studies, the docking protocol used for both approaches was validated by the process of re-docking, preparation of sequence alignment, superimposition, and calculation of root mean square deviation (RMSD) between the original PfDHFR-TS-RJ1 complex and the re-docked PfDHFR-TS-RJ1 complex [43]. The original PfDHFR-TS-RJ1 cocrystallized complex was opened in the Discovery Studio Visualizer software. From this complex, chain $\mathrm{B}$, chain $\mathrm{C}$, chain $\mathrm{D}$, water molecules, and other heteroatoms were removed. Chain A, which comprises the PfDHFR-TS-RJ1 complex, was saved in '.pdb' file format as the original co-crystallized complex.

Re-docking of RJ1 to PfDHFR-TS was carried out on the PyRx 0.8 virtual screening platform and AutoDock Vina stand-alone package with the same parameters and protocols that had been previously described. After docking, the output file (.pdbqt) of RJ1 was opened in a text document with a notepad. The re-docked PfDHFR-TS-RJ1 complex was created by copying all the necessary information on RJ1 and pasting it into the output file (.pdbqt) of PfDHFR-TS, which was also opened in a text document with a notepad. The notepad was saved, and this output file (.pdbqt) was converted into '.pdb' file format with the OpenBabel version 3.1.1. software [44]. This was saved as the re-docked co-crystallized complex.

Both the complexes were loaded onto the Discovery Studio Visualizer software. Among the toolset, superimpose proteins were selected, wherein the proteins were made to superimpose by sequence alignment. The original co-crystallized complex was set as the reference protein. Finally, the RMSD between the two complexes was calculated. This process was done to validate the docking protocol used for both approaches.

\subsection{Visualization and analysis of ligand interactions.}

The phytocompound with the best binding affinity for the active binding site of PfDHFR-TS as computed out from the two different approaches were taken for further studies. Analysis and visualization of the 2-dimensional and 3-dimensional ligand interactions were done with the Discovery Studio Visualizer software. Among the toolset, the receptor-ligand interaction tool of the software was used to visualize the ligand interactions. The ligand interactions between the original co-crystallized complex and the re-docked co-crystallized complexes generated on the PyRx 0.8 virtual screening platform and the AutoDock Vina standalone package were comparatively analyzed against the ligand interactions shown by the 
phytocompound with the best binding affinity for the active binding site of PfDHFR-TS. The 3D binding pose of the phytocompound at the active binding pocket of PfDHFR-TS was also developed with the Discovery Studio Visualizer software.

\subsection{Toxicity study.}

The toxicity of the phytocompound with the best binding affinity for the active binding site of PfDHFR-TS was studied with the DataWarrior v.5.2.1 software [45]. The '.sdf' file format of the phytocompound was loaded onto the software. Toxicity profiles such as mutagenicity, tumorigenic, irritant, and reproductive effectiveness were studied for the phytocompound.

\subsection{Molecular properties, bioavailability score, synthetic accessibility.}

The molecular properties of the phytocompound such as molecular weight, number of heavy atoms, number of heavy aromatic atoms, lipophilicity, hydrogen acceptors, hydrogen donors, molar refractivity, topological polar surface area, WLOGP, and water solubility were calculated with DataWarrior v.5.2.1 software [45] and SwissADME web tool [46].

Adherence of the phytocompound to various principles such as Lipinski's rule of 5, Ghose filter, Veber filter, Egan filter, Meugge filter were also analyzed with the SwissADME web tool. The overall bioavailability score and synthetic accessibility of the phytocompound were predicted with the SwissADME web tool, while the drug score of the phytocompound was computed with DataWarrior v.5.2.1 software.

\subsection{Bioactivity prediction.}

The bioactivity profile of FG17 was predicted with the Molinspiration Chemoinformatics web tool [47] and was compared to those of RJ1 and artemisinin. The SMILES ID of FG17, RJ1, and artemisinin [48] was generated with the MarvinSketch 20.10 software. The web tool was accessed, the SMILES ID was entered in the search box, and the bioactivity profiles were generated.

\section{Results and Discussion}

\subsection{Docking studies using PyRx 0.8.}

PyRx 0.8 software is a virtual screening platform that uses AutoDock Vina to dock ligands to a target protein. AutoDock Vina is more efficient and precise in comparison to the conventional AutoDock [38, 39]. Molecular docking is widely used in the drug discovery process for lead identification and optimization [49]. Binding affinity values of a ligand towards a target protein are generated by the molecular docking simulation studies [50]. The lower the numerical $(\mathrm{kcal} / \mathrm{mol})$ value, the higher the binding affinity of the ligands towards the target proteins [38]. The binding affinities of the RJ1 and the FGs towards the target protein are given in Table 1. Re-docking of the co-crystal inhibitor (RJ1) produced a binding affinity of $-7.9 \mathrm{kcal} / \mathrm{mol}$. On the other hand, with a binding affinity value of $-10.4 \mathrm{kcal} / \mathrm{mol}, \mathrm{FG} 17$ is the phytocompound with the best binding affinity among all the phytocompounds. All the FGs except for FG4 $(-7.5 \mathrm{kcal} / \mathrm{mol})$ and FG6 $(-1.5 \mathrm{kcal} / \mathrm{mol})$ have a better binding affinity towards the target protein in comparison to RJ1. 
From Table 1, it can be observed that there are only minute differences between the binding affinity of different ligands. For example, the differences in the binding affinities between FG17, FG15, FG11, FG8, FG5, and so on are within a single-digit decimal value. Many ligands showed good potential. So, it may be assumed that different ligands showed a good binding affinity for the target protein. On the other hand, the minute differences between the binding affinities of different ligands made it complex to draw a confident conclusion regarding the best phytocompound to be proposed against PfDHFR-TS. Therefore, it was decided to conduct an additional molecular docking simulation study with a more rigorous approach.

Table 1. Binding affinities of FGs towards the active binding site of $P f$ DHFR-TS.

\begin{tabular}{c|c} 
Compound ID & Binding affinity (-kcal/mol) \\
\hline RJ1 & 7.9 \\
\hline FG1 & 10.0 \\
\hline FG2 & 10.0 \\
\hline FG3 & 9.7 \\
\hline FG4 & 7.5 \\
\hline FG5 & 10.1 \\
\hline FG6 & 1.5 \\
\hline FG7 & 9.4 \\
\hline FG8 & 10.2 \\
\hline FG9 & 9.7 \\
\hline FG10 & 9.9 \\
\hline FG11 & 10.2 \\
\hline FG12 & 9.0 \\
\hline FG13 & 9.5 \\
\hline FG14 & 9.2 \\
\hline FG15 & 10.2 \\
\hline FG16 & 9.3 \\
\hline FG17 & 10.4
\end{tabular}

\subsection{Docking studies using AutoDock Vina.}

AutoDockTools1.5.6 offers a simple graphical user interface to prepare proteins and ligands for molecular docking simulation studies [41]. Unlike the first docking simulation, the energy of the target protein was minimized with Schrodinger Maestro. Also, Kollman charges were added to the target protein with AutoDockTools1.5.6. To all the ligands whose energy was minimized, hydrogens and Gasteiger charges were also added with AutoDockTools1.5.6. Finally, molecular docking was carried out with the stand-alone AutoDock Vina package.

The binding affinities of the RJ1 and the FGs towards the target protein are given in Table 2. Re-docking of the co-crystal inhibitor (RJ1) produced a binding affinity of -7.9 $\mathrm{kcal} / \mathrm{mol}$. Both of the docking approaches produced the same binding affinity for RJ1. With a binding affinity value of $-10.8 \mathrm{kcal} / \mathrm{mol}$, FG17 is the phytocompound with the best binding affinity among all the phytocompounds. Also, all the FGs except for FG1 (-7.1 kcal/mol), FG9 $(-4.3 \mathrm{kcal} / \mathrm{mol})$, and FG10 $(-4.4 \mathrm{kcal} / \mathrm{mol})$ have a better binding affinity towards the target protein in comparison to $\mathrm{RJ} 1$.

In the previous simulation on PyRx 0.8 virtual screening platform, we had argued that the differences between the binding affinity value of the FGs were too small and that it was difficult to draw an assertive conclusion. To aid the team in decision-making, we conducted another simulation with a different approach. Although the differences in binding affinity values between some FGs were still small, FG17 still has the best binding affinity among all the phytocompounds. Therefore, we decided to validate the docking protocols of both the docking approaches to ascertain the accuracy of our in-silico work. 
Table 2. Binding affinities of FGs towards the active binding site of PfDHFR-TS

\begin{tabular}{c|c} 
Compound ID & Binding affinity (-kcal/mol) \\
\hline RJ1 & 7.9 \\
\hline FG1 & 7.1 \\
\hline FG2 & 9.4 \\
\hline FG3 & 8.2 \\
\hline FG4 & 10.4 \\
\hline FG5 & 8.6 \\
\hline FG6 & 10.4 \\
\hline FG7 & 8.3 \\
\hline FG8 & 10 \\
\hline FG9 & 4.3 \\
\hline FG10 & 4.4 \\
\hline FG11 & 8.8 \\
\hline FG12 & 9.3 \\
\hline FG13 & 9.6 \\
\hline FG14 & 9.5 \\
\hline FG15 & 10.4 \\
\hline FG16 & 9 \\
\hline FG17 & 10.8
\end{tabular}

\subsection{Validation of docking.}

Validation of docking was carried out to validate the efficiency of the docking procedure. This process was done by re-docking RJ1 to its active binding site, followed by preparation of sequence alignment, superimposition, and RMSD calculation [43]. Re-docking of RJ1 to the active binding site of the protein with the PyRx 0.8 virtual screening platform and the AutoDock Vina stand-alone package was carried out as per the methods previously described. In both the approach, a binding affinity value of $-7.9 \mathrm{kcal} / \mathrm{mol}$ was observed after re-docking. The re-docked PfDHFR-TS-RJ1 complex was superimposed to the original PfDHFR-TS-RJ1 complex using the Discovery Studio Visualizer software.

For the output files obtained from the PyRx 0.8 virtual screening platform, the RMSD between the two complexes was $0.00 \AA$. On the other hand, for AutoDock Vina stand-alone package, the RMSD between the two complexes was $0.259 \AA$. The docking protocol used for both approaches made RJ1 bind to the same active binding site of the target protein after redocking (see Figure 2). A low RMSD value is preferred over a higher value [43, 51]. Other studies had also validated their docking protocol using a similar method. RMSD of $0.615 \AA$, $0.254 \AA$, and $1.94 \AA$ between the original and re-docked complexes had been reported by other studies $[43,51]$.

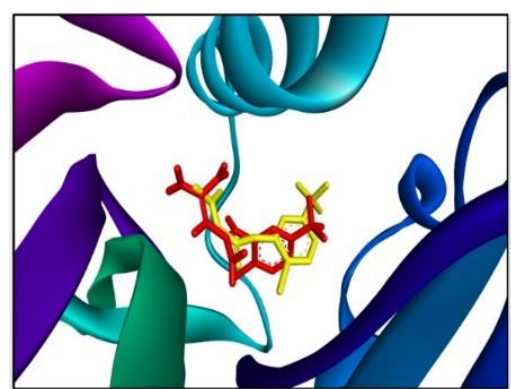

(a)

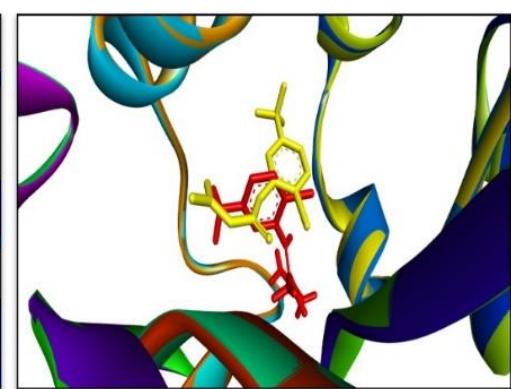

(b)

Figure 2. Superimposed original PfDHFR-TS-RJ1 complex and re-docked PfDHFR-TS-RJ1 complex with (a) PyRx 0.8 tool and (b) AutoDock Vina stand-alone package; RJ1 yellow = original pose at the active binding site; RJ1 red = re-docked pose at the active binding site.

In the original PfDHFR-TS-RJ1 complex, RJ1 interacted with ILE14, ASP54, MET55, PHE58, ILE112, ILE164, and TYR170 (see Figure 3). In the re-docked PfDHFR-TS-RJ1 
complex obtained after docking with the PyRx 0.8 virtual screening platform, RJ1 was found to interact with ILE14, CYS15, ASP54, PHE58, ILE112, LEU119, ILE164, and THR185. Five amino acids such as ILE14, ASP54, PHE58, ILE112, and ILE164 represent the common interacting active site residues between the original complex and the re-docked complex produced by the PyRx 0.8 virtual screening platform (see Figure 3).

On the other hand, in the re-docked PfDHFR-TS-RJ1 complex obtained after docking with the AutoDock Vina stand-alone package, RJ1 was found to interact with ALA16, LEU46, ASP54, PHE58, SER108, ILE112, and ILE164. Four amino acids, such as ASP54, PHE58, ILE112, and ILE164, represent the common interacting active site residues between the original complex and the re-docked complex produced by the AutoDock Vina stand-alone package (see Figure 3). Not only did the docking protocol followed in both approaches allow RJ1 to bind to the same active binding site, but the docked ligands were also able to interact with the majority of the active site residues of the target protein. In this way, the docking protocol for the present study was validated. Among the FGs, FG17 was considered the antimalarial compound (inhibitor) with a promising binding affinity for PfDHFR-TS. Therefore, FG17 was investigated for further studies.

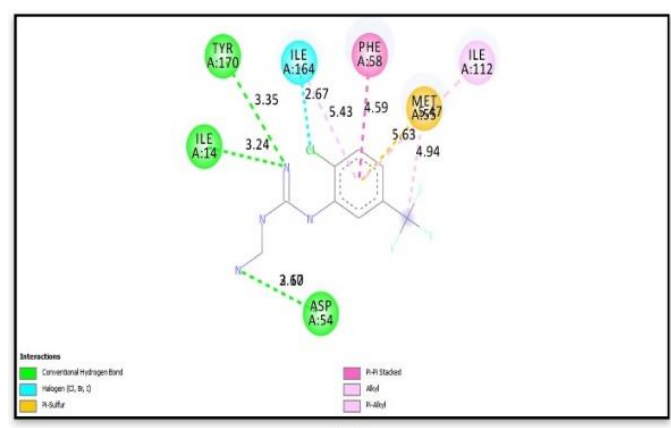

(a)

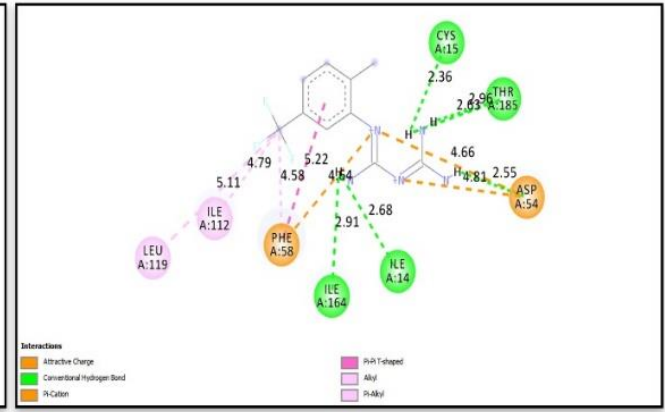

(b)

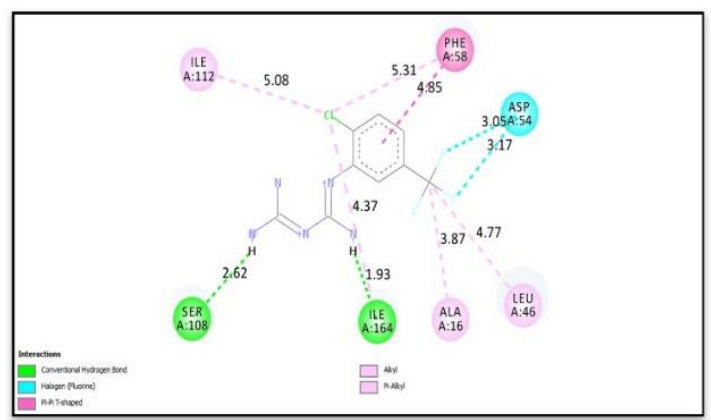

(c)

Figure 3. Ligand interactions between RJ1 and the active site residues in (a) original PfDHFR-TS-RJ1 complex; (b) re-docked PfDHFR-TS-RJ1 complex with PyRx 0.8 tool; (c) re-docked PfDHFR-TS-RJ1 complex with AutoDock Vina stand-alone package

\subsection{Visualization and analysis of ligand interactions.}

In drug development, it is important to understand the ligand interactions that occur between a drug and a protein [52]. The present study analyzed the molecular interactions between PfDHFR-TS with RJ1 and FG17 using the Discovery Studio Visualizer software. The interacting active site residues of PfDHFR-TS with RJ1 and FG17 are summarized in Table 3. FG17 interacted with ALA16, LEU40, GLY41, GLY44, MET55, PHE58, ILE112, LEU119, GLY166, SER167, and TYR170 of PfDHFR-TS. It can be observed that RJ1 (original cocrystallized complex) and FG17 interacted with 4 similar active site residues (MET55, PHE58, 
ILE112, and TYR170). The RJ1 (re-docked with PyRx 0.8 virtual screening tool) and FG17 interacted with 3 similar active site residues (PHE58, ILE112, and LEU119). The RJ1 (redocked with AutoDock Vina stand-alone package) and FG17 interacted with 3 similar active site residues (ALA16, PHE58, and ILE112). The ligand interactions are also represented in 2D and $3 \mathrm{D}$ views (see Figure 4). The 3D binding pose of all the ligands superimposed at the active binding site of PfDHFR-TS is also given in Figure 4.

Table 3. Summary of the interacting active site amino acid residues

\begin{tabular}{l|l} 
Compound ID & Interacting active site residues \\
\hline RJ1 (original complex) & ILE14, ASP54, MET55, PHE58, ILE112, ILE164, TYR170, \\
\hline RJ1 (re-docked complex) $)^{*}$ & ILE14, CYS15, ASP54, PHE58, ILE112, LEU119, ILE164, THR185, \\
\hline RJ1 (re-docked complex) & ALA16, LEU46, ASP54, PHE58, SER108, ILE112, ILE164, \\
\hline FG17* & ALA16, LEU40, GLY41, GLY44, MET55, PHE58, ILE112, \\
& LEU119, GLY166, SER167, TYR170 \\
\hline FG17\# & $\begin{array}{l}\text { ALA16, LEU40, GLY41, GLY44, MET55, PHE58, ILE112, } \\
\text { LEU119, GLY166, SER167, TYR170 }\end{array}$ \\
*PyRx 0.8 virtual screening tool; \#AutoDock Vina stand-alone package
\end{tabular}

"PyRx 0.8 virtual screening tool; \#AutoDock Vina stand-alone package

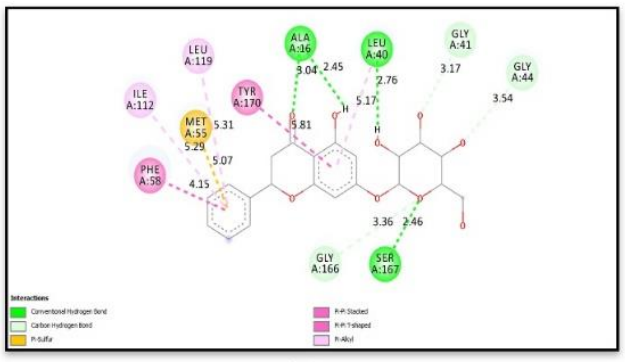

(a)

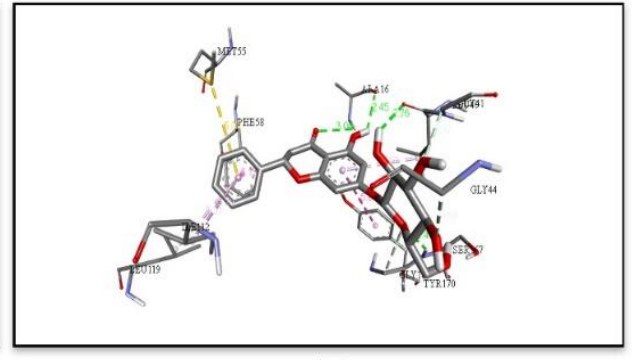

(b)

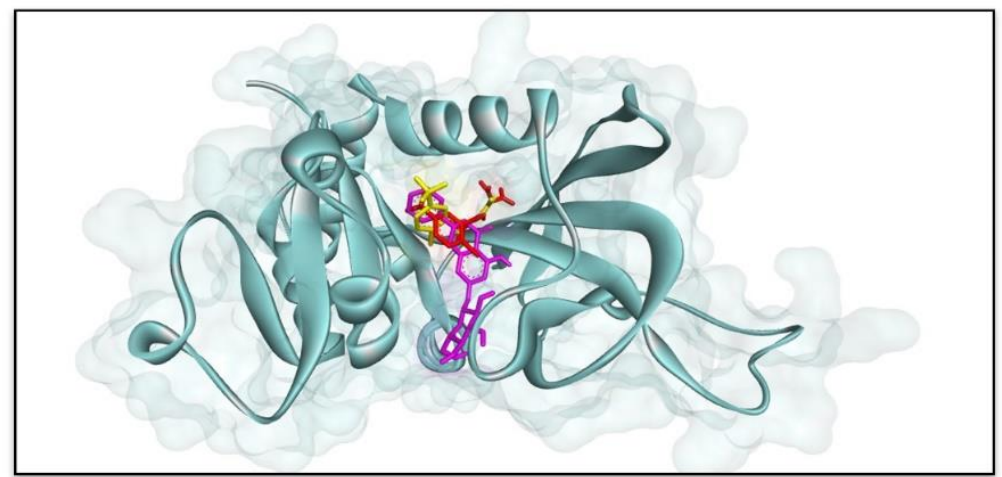

(c)

Figure 4. Visualization of (a) 2D ligand interactions and (b) 3D ligand interactions of FG17 at the active binding site of PfDHFR-TS; (c) Superimposition of the binding poses of RJ1 (yellow color) of the original cocrystallized complex, RJ1 (red color) re-docked with PyRx 0.8 tool and FG17 (purple color) docked with PyRx 0.8 tool.

The in-vitro antimalarial activity of several compounds had been investigated. A handful of those compounds had also been subjected to molecular docking, and their ligand interactions had been analyzed [53-55]. In the present study, we have found that FG17 interacted with many amino acid residues at the active binding site of the protein (see Table 3 ). Therefore, it will be interesting to compare the ligand interactions of FG17 with the ligand interactions shown by compounds having in-vitro antimalarial activity. The comparative analysis of our study's findings with other research works is given in Table 4, wherein compounds reported to interact with similar active site residues were identified and analyzed [53-55]. The alphabet ' $\mathrm{X}$ ' is marked next to the common interacting active site residues (Table 4). Many compounds with in-vitro antimalarial activity interacted with the amino acid residues 
that interacted with FG17 [53-55]. This evidence supports the results obtained from the molecular docking simulation studies.

Table 4. Ligand interactions of FG17 in comparison with compounds having in-vitro antimalarial activity.

\begin{tabular}{|c|c|c|c|c|c|c|c|c|c|}
\hline \multirow{2}{*}{$\begin{array}{c}\text { Amino } \\
\text { acids }\end{array}$} & \multicolumn{9}{|c|}{$\leftarrow$ Compounds $\rightarrow$} \\
\hline & FG17 & CMP1 & CMP2 & CMP3 & CMP4 & CMP5 & CMP6 & CMP7 & CMP8 \\
\hline ALA16 & $X$ & - & - & - & $X$ & $X$ & - & - & - \\
\hline LEU40 & $\mathrm{X}$ & - & - & - & $\mathrm{X}$ & $\mathrm{X}$ & - & - & $\mathrm{X}$ \\
\hline GLY41 & $\mathrm{X}$ & $\mathrm{X}$ & - & - & $\mathrm{X}$ & - & - & - & - \\
\hline GLY44 & $\mathrm{X}$ & - & - & $\mathrm{X}$ & $\mathrm{X}$ & - & - & - & - \\
\hline MET55 & $\mathrm{X}$ & - & - & - & $X$ & $\mathrm{X}$ & $\mathrm{X}$ & $\mathrm{X}$ & $\mathrm{X}$ \\
\hline PHE58 & $\mathrm{X}$ & - & $\mathrm{X}$ & - & $X$ & $\mathrm{X}$ & $\mathrm{X}$ & - & $\mathrm{X}$ \\
\hline ILE112 & $\mathrm{X}$ & $\mathrm{X}$ & $X$ & $\mathrm{X}$ & - & $\mathrm{X}$ & $\mathrm{X}$ & $X$ & $\mathrm{X}$ \\
\hline LEU119 & $\mathrm{X}$ & - & - & - & - & $\mathrm{X}$ & $\mathrm{X}$ & $\mathrm{X}$ & $\mathrm{X}$ \\
\hline GLY166 & $\mathrm{X}$ & $\mathrm{X}$ & - & - & - & - & - & - & - \\
\hline SER167 & $\mathrm{X}$ & - & - & - & - & - & - & - & - \\
\hline TYR170 & $\mathrm{X}$ & - & - & - & - & $X$ & - & $\mathrm{X}$ & - \\
\hline
\end{tabular}

yl)amino)ethyl)piperazine-1-carbothioamide; CMP2 = N-(4-amino-6-((4-methoxyphenyl)amino)-1,3,5triazin-2-yl)-4-(2-((7-chloroquinolin-4- yl)amino)ethyl)piperazine-1-carbothioamide; CMP3 = N-(4-amino6-(o-tolylamino)-1,3,5-triazin-2-yl)-4-(2-((7-chloroquinolin-4- yl)amino)ethyl)piperazine-1-carbothioamide [53]; CMP4 = hybrid 4- aminoquinoline-1,3,5-triazine derivatives [54]; CMP5 (1e), CMP6 (1c), CMP7 (1d), CMP8 (1f) = 4-aminoquinoline-clubbed 1,3,5-triazine derivatives [55]

\subsection{Toxicity profile.}

Toxicity is a major contributing factor to the high cost of drug development. It is one of the many reasons why many therapeutically potent drugs had to be withdrawn from the late clinical study or the market $[56,57]$. Drugs withdrawn from the market are associated with carcinogenicity, cardiovascular toxicity, gastrointestinal toxicity, dermatological toxicity, hematological toxicity, neurological toxicity, ophthalmic toxicity, renal toxicity, respiratory toxicity, and reproductive toxicity [58]. Between 1953 and 2013, 462 drugs were reported to be withdrawn from the market [59]. Therefore, it is important to study the toxicity profile of a compound before conducting further studies and avoiding problems in the future. The toxicity profile of FG17 was studied with Data Warrior v.5.2.1 software. Toxicities such as mutagenicity, tumorigenic, reproductive effectiveness, and irritant of FG17 were studied. From the study, it was found that FG17 did not show any form of toxicity issues. Although FG15 also has a high binding affinity for PfDHFR-TS, it was computed to be mutagenic. A compound with a high binding affinity value does not mean that it will be developed into a lead compound. It only signifies that the phytocompound may be taken for further studies. This validates the importance of studying the toxicity profile of a phytocompound.

\subsection{Molecular properties, bioavailability score, and synthetic accessibility.}

The molecular properties, bioavailability score, and synthetic accessibility of FG17 are given in Table 5. Although FG4, FG5, and FG11 were also found to have a high binding affinity to PfDHFR-TS (see Table 1 and 2), their molecular properties, bioavailability score, and synthetic accessibility score were not better than FG17. To provide a better picture, these parameters of FG4, FG5, and FG11 are also included in Table 5. Although a compound might have a good binding affinity for a target protein, it does not mean that it is suitable to be the lead compound. This validates the importance of carrying out an in-depth study on a compound. 
Lipinski's rule of 5 (Lro5) [60] states that a drug is considered bioavailable if its molecular weight, cLogP, H-A, and H-D are $\leq 500,5,10$, and 5, respectively. According to Lro5, FG17 did not violate any rules and had zero violations. FG4, FG5, and FG11 violated at least one rule of Lro5.

TPSA is the sum of all the surface areas covered by polar atoms in a compound. Molecular property such as TPSA is a parameter that is often considered as important in the drug discovery process [61]. Ideally, a low TPSA value is preferred over a higher value for a potential lead candidate. According to the Veber filter, FG17 has 1 violation as the TPSA is greater than $140 \AA^{2}$ [62]. According to the Egan filter, FG17 also has 1 violation as the TPSA is greater than $131.6 \AA^{2}$ [63]. The TPSA of FG4 (181.05 $\left.\AA^{2}\right)$, FG5 (289.66 $\left.\AA^{2}\right)$, and FG11 $\left(190.28 \AA^{2}\right)$ are all higher than that of FG17 (145.91 $\left.\AA^{2}\right)$.

Water solubility is an important parameter for drugs that are intended to be administered orally. Poorly soluble drugs in water often lead to low bioavailability, including gastrointestinal toxicity [64]. FG17 was found to have good solubility in water. According to the Ghose filter, FG17 showed no violation (Mol. Wt.>480, MR>130, WLOGP<-0.4, a number of atoms $>70$ ) [65]. According to Muegge filter, FG17 showed no violation (Mol. Wt.>600, TPSA > 150, HA $>10$, H-D > 5) [66].

The pace of the drug discovery process is often slowed down by bioavailability issues $[67,68]$. Many compounds of natural origin, such as flavonoids, often have bioavailability issues [69]. It is important to predict the bioavailability of a compound to avoid problems in the future. FG17 has a good bioavailability score of 0.55 . The high bioavailability score of FG17 can also be correlated to its molecular properties. Also, FG17 did not violate Lro5. FG4 also has a bioavailability score of 0.55 , while FG5 and FG11 have a low bioavailability score of 0.17 .

Synthetic accessibility gives an idea of the degree of difficulty it will take to synthesize a compound by providing a numerical value as a reference. A score of 1 suggests that the compound will be easy to synthesize, while a score of 10 indicates that the compound will be difficult to synthesize [46]. FG17 has an average synthetic accessibility score of 4.94. On the other hand, FG4 (4.99), FG5 (6.56), and FG11 (5.21) have a high synthetic accessibility score implying that they will be difficult to synthesize.

Table 5. Molecular properties, bioavailability score, and synthetic accessibility

\begin{tabular}{c|c|c|c|c} 
Properties & FG17 & FG4 & FG5 & FG11 \\
\hline Molecular weight & 418.397 & 432.38 & 626.52 & 448.38 \\
\hline Number of Heavy atoms & 30 & 31 & 44 & 32 \\
\hline Number of Aromatic Heavy Atoms & 12 & 16 & 16 & 16 \\
\hline cLogP & 0.5121 & -0.05 & -2.18 & -0.15 \\
\hline Hydrogen acceptors & 9 & 10 & 17 & 11 \\
\hline Hydrogen donors & 5 & 7 & 11 & 7 \\
\hline Molar refractivity & 101.67 & 106.61 & 142.54 & 108.13 \\
\hline Topological polar surface area & $145.91 \AA^{2}$ & $181.05 \AA^{2}$ & $289.66 \AA^{2}$ & $190.28 \AA^{2}$ \\
\hline WLOGP & -0.05 & -0.23 & -2.71 & -0.04 \\
\hline Water solubility & Soluble & Soluble & Soluble & Soluble \\
\hline Lipinski's rule of 5 & 0 violation & 1 violation & 3 violations & 2 violations \\
\hline Ghose filter & 0 violation & 0 violation & 4 violations & 0 violation \\
\hline Veber filter & 1 violation & 1 violation & 1 violation & 1 violation \\
\hline Egan filter & 1 violation & 1 violation & 1 violation & 1 violation \\
\hline Muegge filter & 0 violation & 2 violations & 4 violations & 3 violations \\
\hline Bioavailability score & 0.55 & 0.55 & 0.17 & 0.17 \\
\hline Synthetic accessibility & 4.94 & 4.99 & 6.56 & 5.21
\end{tabular}




\subsection{Bioactivity prediction.}

GPCRs serve as a favorable drug target as they regulate cell signaling, stimuli responses, and cellular homeostasis processes [70]. Several ion channels have pharmacologically favorable binding sites. These binding sites may be exploited therapeutically as the function of ion channels can be modulated in multiples ways [71]. Kinases play an important role in cellular signaling, and hence they are one of the most favorable targets, especially in the field of anti-cancer drug discovery [72].

Nuclear receptors are transcription factors that ligands can induce. They are an attractive drug target as they are actively involved in physiological processes and are also associated with many diseases [73]. Proteases are clinically important drug targets as they are associated with many viral diseases. Inhibition of a viral protease prevents the replication of viral RNA [74]. Enzymes are an important class of drug targets that have been increasingly employed in computer-aided drug design to identify lead compounds. Many compounds inhibit enzymes in their specific way, and this principle is extensively used in the drug discovery process [75].

The bioactivity of FG17 against GPCRs, ion channels, kinases, nuclear receptors, protease, and enzyme was predicted using the Molinspiration Chemoinformatics web tool (Table 6). The predicted bioactivity of FG17 was compared with RJ1 and artemisinin. Based on the score, the bioactivity was categorized as inactive $(<-0.5)$, moderately active $(-0.5$ to 0$)$, and as having a considerable biological activity ( $>0)$ [76]. Dihydrofolate reductasethymidylate synthase (DHFR-TS) is a bifunctional enzyme essential for the folate biosynthesis in P. falciparum [23]. FG17 was predicted as a better enzyme inhibitor than RJ1 and artemisinin. Overall, FG17 showed a better bioactivity profile as compared to RJ1 and artemisinin.

Table 6. Predicted bioactivity of C_17 and hesperetin in comparison with CCI and artemisinin.

\begin{tabular}{c|c|c|c|c|c|c} 
Compound & GPCR & ICM & KI & NRL & PI & EI \\
\hline FG17 & 0.16 & -0.09 & -0.15 & 0.31 & 0.10 & 0.40 \\
\hline RJ1 & 0.06 & 0.39 & -0.26 & -0.86 & 0.07 & -0.03 \\
\hline Artemisinin & -0.17 & -0.31 & -0.65 & -0.00 & -0.19 & 0.39
\end{tabular}

$\mathrm{GPCR}=\mathrm{G}$ protein coupled receptor; $\mathrm{ICM}=$ Ion channel modulator; $\mathrm{KI}=$ Kinase inhibitor; NRL = Nuclear receptor ligand; PI = Protease inhibitor; EI = Enzyme inhibitor

\section{Conclusions}

The study concludes that FG17 (pinocembrin-7-O- $\beta$-D-glucopyranoside) of A. pennata is the most promising flavonoid-glycoside effective against PfDHFR-TS. However, further experimental (in vitro/ in vivo) studies are required to fully understand the activity of FG17 against PfDHFR-TS. The findings of our present investigation may serve as an attractive strategy to develop newer and safer antimalarial compounds using the novel flavonoid scaffold of pinocembrin.

\section{Funding}

This research received no external funding. 


\section{Acknowledgments}

The corresponding author (J.H.Z.) acknowledges Prof. H. Lalthanzara, Department of Zoology, Pachhunga University College, for his valuable technical advice and moral support.

\section{Conflicts of Interest}

The authors declare no conflict of interest.

\section{References}

1. World malaria report 2020: 20 years of global progress and challenges. Available online: https://www.who.int/publications/i/item/9789240015791 (accessed on 9 April 2021).

2. da Silva, M.A.; Veloso, M.P.; de Souza Reis, K.; de Matos Passarini, G.; Dos Santos, A.P.A.; do Nascimento Martinez, L.; Fokoue, H.H.; Kato, M.J.; Teles, C.B.G.; Kuehn, C.C. In silico evaluation and in vitro growth inhibition of Plasmodium falciparum by natural amides and synthetic analogs. Parasitol Res 2020, 119, 18791887, https://doi.org/10.1007/s00436-020-06681-9.

3. Luzolo, A.L.; Ngoyi, D.M. Cerebral malaria. Brain Res Bull 2019, 145, 53-58, https://doi.org/10.1016/j.brainresbull.2019.01.010.

4. Garrido-Cardenas, J. A.; Cebrián-Carmona, J.; González-Cerón, L.; Manzano-Agugliaro, F.; Mesa-Valle, C. Analysis of Global Research on Malaria and Plasmodium vivax. Int J Environ Res Public Health 2019, 16, https://doi.org/10.3390/ijerph16111928.

5. Mackintosh, C.L.; Beeson, J.G.; Marsh, K. Clinical features and pathogenesis of severe malaria. Trend. Parasitol 2004, 20, 597-603, https://doi.org/10.1016/j.pt.2004.09.006.

6. Dasgupta, T.; Chitnumsub, P.; Kamchonwongpaisan, S.; Maneeruttanarungroj, C.; Nichols, S.E.; Lyons, T.M.; Tirado-Rives, J.; Jorgensen, W.L.; Yuthavong, Y.; Anderson, K.S. Exploiting structural analysis, in silico screening, and serendipity to identify novel inhibitors of drug-resistant falciparum malaria. ACS Chem Biol 2009, 4, 29-40, https://doi.org/10.1021/cb8002804.

7. Cui, L.; Mharakurwa, S.; Ndiaye, D.; Rathod, P.K.; Rosenthal, P.J. Antimalarial drug resistance: Literature review and activities and findings of the ICEMR network. Am J Trop Med Hyg 2015, 93, 57-68, https://doi.org/10.4269/ajtmh.15-0007.

8. Gachelin, G.; Garner, P.; Ferroni, E.; Verhave, J.P.; Opinel, A. Evidence and strategies for malaria prevention and control: a historical analysis. Malar J 2018, 17, https://doi.org/10.1186/s12936-018-2244-2.

9. Memvanga, P.B.; Tona, G.L.; Mesia, G.K.; Lusakibanza, M.M.; Cimanga, R.K. Antimalarial activity of medicinal plants from the Democratic Republic of Congo: A review. J Ethnopharmacol 2015, 169, 76-98, https://doi.org/10.1016/j.jep.2015.03.075.

10. Okell, L.C.; Griffin, J.T.; Roper, C. Mapping sulphadoxine-pyrimethamine-resistant Plasmodium falciparum malaria in infected humans and in parasite populations in Africa. Sci Rep 2017, 7, https://doi.org/10.1038/s41598-017-06708-9.

11. Menard, D.; Dondorp, A. Antimalarial drug resistance: a threat to malaria elimination. Cold Spring Harb Perspect Med 2017, 7, https://doi.org/10.1101/cshperspect.a025619.

12. Hussien, M.; Abdel Hamid, M.M.; Elamin, E.A.; Hassan, A.O.; Elaagip, A.H.; Salama, A.; Abdelraheem, M.H.; Mohamed, A.O. Antimalarial drug resistance molecular makers of Plasmodium falciparum isolates from Sudan during 2015-2017. PloS one 2020, 15, https://doi.org/10.1371/journal.pone.0235401.

13. Ippolito, M.M.; Moser, K.A.; Kabuya, J.B.; Cunningham, C., Juliano, J.J. Antimalarial Drug Resistance and Implications for the WHO Global Technical Strategy. Curr Epidemiol Rep 2021, 8, 46-62, https://doi.org/10.1007/s40471-021-00266-5.

14. Zhao, D.; Zhang, H.; Ji, P.; Li, S.; Yang, C; Liu, Y.; Qian, D.; Deng, Y.; Wang, H.; Lu, D.; Zhou, R.; Zhao, Y. Surveillance of Antimalarial Drug-Resistance Genes in Imported Plasmodium falciparum Isolates From Nigeria in Henan, China, 2012-2019. Front Cell Infect Microbiol 2021, 11, https://doi.org/10.3389/fcimb.2021.644576.

15. Malaria. Available online: https://www.who.int/news-room/fact-sheets/detail/malaria (accessed on 9 April 2021).

16. Nweze, J.A.; Mbaoji, F.N.; Li, Y.M.; Yang, L.Y.; Huang, S.S.; Chigor, V.N.; Eze, E.A.; Pan, L.X.; Zhang, T., Yang, D.F. Potentials of marine natural products against malaria, leishmaniasis, and trypanosomiasis parasites: a review of recent articles. Infect Dis Poverty 2021, 10, https://doi.org/10.1186/s40249-021-007966.

17. Tisnerat, C.; Dassonville-Klimpt, A.; Gosselet, F.; Sonnet, P. Antimalarial drug discovery: from quinine to the most recent promising clinical drug candidates. Curr Med Chem 2021, https://doi.org/10.2174/0929867328666210803152419. 
18. van der Pluijm, R.W.; Amaratunga, C.; Dhorda, M.; Dondorp, A.M. Triple Artemisinin-Based Combination Therapies for Malaria - A New Paradigm?. Trends Parasitol 2021, 37, 15-24, https://doi.org/10.1016/j.pt.2020.09.011.

19. Memvanga, P.B.; Tona, G.L.; Mesia, G.K.; Lusakibanza, M.M.; Cimanga, R.K. Antimalarial activity of medicinal plants from the Democratic Republic of Congo: A review. J Ethnopharmacol 2015, 169, 76-98, https://doi.org/10.1016/j.jep.2015.03.075.

20. Ntie-Kang, F.; Amoa, O.P.; Lifongo, L.L.; Ndom, J.C.; Sippl, W.; Mbaze, L.M. The potential of antimalarial compounds derived from African medicinal plants, part II: a pharmacological evaluation of non-alkaloids and nonterpenoids. Malar J 2014, 13, https://doi.org/10.1186/1475-2875-13-81.

21. Barmade, M.A.; Murumkar, P.R.; Sharma, M.K.; Shingala, K.P.; Giridhar, R.R.; Yadav, M.R. Discovery of antimalarial agents through application of in silico studies. Comb Chem High Throughput Screen 2015, 18, 151-187, https://doi.org/10.2174/1386207318666141229125852.

22. Joseph Sahayarayan, J.; Soundar Rajan, K.; Nachiappan, M.; Prabhu, D.; Guru Raj Rao, R.; Jeyakanthan, J.; Hossam Mahmoud, A.; Mohammed, O.B.; Morgan, A.M.A. Identification of potential drug target in malarial disease using molecular docking analysis. Saudi J Biol Sci 2020, 27, 3327-3333, https://doi.org/10.1016/j.sjbs.2020.10.019.

23. Chaianantakul, N.; Sungkapong, T.; Supatip, J.; Kingsang, P.; Kamlaithong, S.; Suwanakitti, N. Antimalarial effect of cell penetrating peptides derived from the junctional region of Plasmodium falciparum dihydrofolate reductase-thymidylate synthase. Peptides 2020, 131, https://doi.org/10.1016/j.peptides.2020.170372.

24. Ittarat, W.; Pornthanakasem, W.; Mungthin, M.; Suwandittakul, N.; Leelayoova, S.; Tarnchompoo, B.; Yuthavong, Y.; Kongkasuriyachai, D.; Leartsakulpanich, U. Characterization of Plasmodium knowlesi dihydrofolate reductase-thymidylate synthase and sensitivity to antifolates. Parasitol Int 2018, 67, 787-792, https://doi.org/10.1016/j.parint.2018.08.004.

25. Gogoi, N.; Chetia, D.; Gogoi, B.; Das, A. Multiple-targets Directed Screening of Flavonoid Compounds from Citrus Species to find out Antimalarial Lead with Predicted Mode of Action: An In Silico and Whole Cellbased In vitro Approach. Curr Comput-aided Drug Des 2021, 17, 69-82, https://doi.org/10.2174/1573409916666191226103000.

26. Fang, J.; Liu, C.; Wang, Q.; Lin, P.; Cheng, F. In silico polypharmacology of natural products. Brief Bioinform 2018, 19, 1153-1171, https://doi.org/10.1093/bib/bbx045.

27. Laldinsanga; Sarma, H.; Jahan, T.; Goswami, A.K.; Sharma, R.; Sharma, H.K. Traditional antimalarial drugs from Serchhip and Lunglei districts of Mizoram. Curr Trends Pharm Res 2019, 6, 76-104.

28. Dongmo, A.B.; Miyamoto, T.; Yoshikawa, K.; Arihara, S.; Lacaille-Dubois, M.A. Flavonoids from Acacia pennata and their cyclooxygenase (COX-1 and COX-2) inhibitory activities. Planta Med 2007, 73, 12021207, https://doi.org/10.1055/s-2007-981596.

29. Kim, A.; Choi, J.; Htwe, K.M.; Chin, Y.W.; Kim, J.; Yoon, K.D. Flavonoid glycosides from the aerial parts of Acacia pennata in Myanmar. Phytochemistry 2015, 118, 17-22, https://doi.org/10.1016/j.phytochem.2015.08.001.

30. Rifai, Y.; Arai, M.A.; Koyano, T.; Kowithayakorn, T.; Ishibashi, M. Terpenoids and a flavonoid glycoside from Acacia pennata leaves as hedgehog/GLI-mediated transcriptional inhibitors. J Nat Prod 2010, 73, 995997, https://doi.org/10.1021/np1000818.

31. Chepkirui, C.; Ochieng, P. J.; Sarkar, B.; Hussain, A.; Pal, C.; Yang, L.J.; Coghi, P.; Akala, H.M.; Derese, S.; Ndakala, A.; Heydenreich, M.; Wong, V.; Erdélyi, M.; Yenesew, A. Antiplasmodial and antileishmanial flavonoids from Mundulea sericea. Fitoterapia 2021, 149, https://doi.org/10.1016/j.fitote.2020.104796.

32. Magozwi, D. K.; Dinala, M.; Mokwana, N.; Siwe-Noundou, X.; Krause, R.; Sonopo, M.; McGaw, L.J.; Augustyn, W.A.; Tembu, V.J. Flavonoids from the Genus Euphorbia: Isolation, Structure, Pharmacological Activities and Structure-Activity Relationships. Pharmaceuticals 2021, 14 , https://doi.org/10.3390/ph14050428.

33. Ovenden, S.P.; Cobbe, M.; Kissell, R.; Birrell, G.W.; Chavchich, M.; Edstein, M.D. Phenolic glycosides with antimalarial activity from Grevillea "Poorinda Queen". $J$ Nat Prod 2011, 74, 74-78, https://doi.org/10.1021/np100737q.

34. Graziose, R.; Grace, M.H.; Rathinasabapathy, T.; Rojas-Silva, P.; Dekock, C.; Poulev, A.; Lila, M.A.; Smith, P.; Raskin, I. Antiplasmodial activity of cucurbitacin glycosides from Datisca glomerata (C. Presl) Baill. Phytochemistry 2013, 87, 78-85, https://doi.org/10.1016/j.phytochem.2012.11.025.

35. RCSB Protein Data Bank. Available online: https://www.rcsb.org/structure/3DGA (accessed 5 April 2021).

36. Discoverty Studio Visualizer. Available online: https://discover.3ds.com/discovery-studio-visualizerdownload (accessed 28 December 2020).

37. ChemAxon Marvin. Available online: https://chemaxon.com/products/marvin (accessed 15 May 2020).

38. Trott, O.; Olson, A.J. AutoDock Vina: improving the speed and accuracy of docking with a new scoring function, efficient optimization, and multithreading. $J$ Comput Chem 2010, 31, 455-461, https://doi.org/10.1002/jcc.21334.

39. PyRx. Available online: https://pyrx.sourceforge.io/ (accessed 5 January 2021).

40. Dallakyan, S.; Olson, A.J. Small-molecule library screening by docking with PyRx. Methods Mol Biol 2015, 1263, 243-250, https://doi.org/10.1007/978-1-4939-2269-7_19. 
41. AutoDock. Available online: http://autodock.scripps.edu/resources/adt (accessed 25 July 2021).

42. Maestro, Schrödinger, LLC, New York, NY, 2021. Available online: https://www.schrodinger.com/products/maestro (accessed 2 August 2021).

43. Shivanika, C.; Deepak Kumar, S.; Ragunathan, V.; Tiwari, P.; Sumitha, A.; Brindha Devi, P. Molecular docking, validation, dynamics simulations, and pharmacokinetic prediction of natural compounds against the SARS-CoV-2 main-protease. J Biomol Struct Dyn 2020, 8, 1-27, https://doi.org/10.1080/07391102.2020.1815584.

44. Open Babel. Available online: https://sourceforge.net/projects/openbabel/ (accessed 24 April 2021).

45. Sander, T.; Freyss, J.; von Korff, M.; Rufener, C. DataWarrior: An Open-Source Program For Chemistry Aware Data Visualization And Analysis. J Chem Inf Model 2015, 55, 460-473, https://doi.org/10.1021/ci500588j.

46. Daina, A.; Michielin, O.; Zoete, V. SwissADME: a free web tool to evaluate pharmacokinetics, drug-likeness and medicinal chemistry friendliness of small molecules. Sci Rep 2017, 7, https://doi.org/10.1038/srep42717.

47. Molinspiration Chemoinformatics. Available online: https://www.molinspiration.com/ (accessed 3 August 2021).

48. Artemisinin. Available online: https://pubchem.ncbi.nlm.nih.gov/compound/Artemisinin (accessed 3 August 2021).

49. Pinzi, L.; Rastelli, G. Molecular docking: Shifting paradigms in drug discovery. Int J Mol Sci 2019, 20, https://doi.org/10.3390/ijms20184331.

50. Jiao, X.; Jin, X.; Ma, Y.; Yang, Y.; Li, J.; Liang, L.; Liu, R.; Li, Z. A comprehensive application: Molecular docking and network pharmacology for the prediction of bioactive constituents and elucidation of mechanisms of action in component-based Chinese medicine. Comput Biol Chem 2021, 90, https://doi.org/10.1016/j.compbiolchem.2020.107402.

51. Gentile, D.; Patamia, V.; Scala, A.; Sciortino, M.T.; Piperno, A.; Rescifina, A. Putative inhibitors of SARSCoV-2 main protease from a library of marine natural products: A virtual screening and molecular modeling study. Mar Drugs 2020, 18, https://doi.org/10.3390/md18040225.

52. Anighoro, A. Underappreciated Chemical Interactions in Protein-Ligand Complexes. Met Mol Biol 2020, 2114, 75-86, https://doi.org/10.1007/978-1-0716-0282-9_5.

53. Bhat, H.R.; Singh, U.P.; Thakur, A.; Ghosh, S.K.; Gogoi, K.; Prakash, A.; Singh R.K. Synthesis, antimalarial activity and molecular docking of hybrid 4-aminoquinoline-1,3,5-triazine derivatives. Exp Parasitol 2015, 157, 59-67, https://doi.org/10.1016/j.exppara.2015.06.016.

54. Bhat, H.R.; Singh, U.P.; Gahtori, P.; Ghosh, S.K.; Gogoi, K.; Prakash, A.; Singh, R.K. Synthesis, Docking, In Vitro and In Vivo Antimalarial Activity of Hybrid 4-aminoquinoline-1,3,5-triazine Derivatives Against Wild and Mutant Malaria Parasites. Chem Biol Drug Des 2015, 86, 265-271, https://doi.org/10.1111/cbdd.12490.

55. Bhat, H.R.; Ghosh, S.K.; Prakash, A.; Gogoi, K.; Singh, U.P. In vitro antimalarial activity and molecular docking analysis of 4-aminoquinoline-clubbed 1,3,5-triazine derivatives. Lett Appl Microbiol 2012, 54, 483486, https://doi.org/10.1111/j.1472-765X.2012.03234.x.

56. Agustí, A.; Aguilera, C.; Bosch, M.; Danés, I.; Pérez, E.; Vendrell, L.; Aller, M.B.; Boixareu, N.; GarcíaDoladé, N.; Diogène, E. Withdrawal of hospital outpatient treatments in severe diseases due to unacceptable toxicity: A retrospective study from the register of patients and treatments. Br J Clin Pharmacol 2021, 87, 2549-2557, https://doi.org/10.1111/bcp.14665.

57. Guengerich, F.P. Mechanisms of drug toxicity and relevance to pharmaceutical development. Drug Metab Pharmacokinet 2011, 26, 3-14, https://doi.org/10.2133/dmpk.dmpk-10-rv-062.

58. Siramshetty, V.B.; Nickel, J.; Omieczynski, C.; Gohlke, B.O.; Drwal, M.N.; Preissner, R. WITHDRAWN-a resource for withdrawn and discontinued drugs. Nucleic Acids Res 2016, 44, 1080-1086, https://doi.org/10.1093/nar/gkv1192.

59. Onakpoya, I.J.; Heneghan, C.J.; Aronson, J.K. Post-marketing withdrawal of 462 medicinal products because of adverse drug reactions: a systematic review of the world literature. BMC Med 2016, 14, https://doi.org/10.1186/s12916-016-0553-2.

60. McKerrow, J.H.; Lipinski, C.A. The rule of five should not impede anti-parasitic drug development. Int $J$ Parasitol Drugs Drug Resist 2017, 7, 248-249, https://doi.org/10.1016/j.ijpddr.2017.05.003.

61. Zhong, H.A.; Mashinson, V.; Woolman, T.A.; Zha, M. Understanding the molecular properties and metabolism of top prescribed drugs. Curr Top Med Chem 2013, 13, 1290-1307, https://doi.org/10.2174/15680266113139990034.

62. Veber, D.F.; Johnson, S.R.; Cheng, H.Y.; Smith, B.R.; Ward, K.W.; Kopple, K.D. Molecular properties that influence the oral bioavailability of drug candidates. J Med Chem 2002, 45, 2615-2623, https://doi.org/10.1021/jm020017n.

63. Egan, W.J.; Merz, K.M.; Baldwin, J.J. Prediction of drug absorption using multivariate statistics. J Med Chem 2000, 43, 3867-3877, https://doi.org/10.1021/jm000292e.

64. Pal, S.; Mehta, D.; Dasgupta, U.; Bajaj, A. Advances in engineering of low molecular weight hydrogels for chemotherapeutic applications. Biomed Mater 2021, 16, https://doi.org/10.1088/1748-605X/abdce1. 
65. Ghose, A.K.; Viswanadhan, V.N.; Wendoloski, J.J. A knowledge-based approach in designing combinatorial or medicinal chemistry libraries for drug discovery. 1. A qualitative and quantitative characterization of known drug databases. J Comb Chem 1999, 1, 55-68, https://doi.org/10.1021/cc9800071.

66. Muegge, I.; Heald, S.L.; Brittelli, D. Simple selection criteria for drug-like chemical matter. J Med Chem 2001, 44, 1841-1846, https://doi.org/10.1021/jm015507e.

67. Jansook, P.; Hnin, H.M.; Loftsson, T.; Stefánsson, E. Cyclodextrin-based formulation of carbonic anhydrase inhibitors for ocular delivery - A review. Int $J$ Pharm 2021, 606, https://doi.org/10.1016/j.ijpharm.2021.120955.

68. Han, M.; Wang, Y.; Cook, K.; Bala, N.; Soto, M.; Rock, D.A.; Pearson, J.T.; Rock, B.M. Universal Automated Immunoaffinity Purification-CE-MS Platform for Accelerating Next Generation Biologic Design. Anal Chem 2021, 93, 5562-5569, https://doi.org/10.1021/acs.analchem.1c00149.

69. Zhao, J.; Yang, J.; Xie Y. Improvement strategies for the oral bioavailability of poorly water-soluble flavonoids: An overview. Int J Pharm 2019, 570, https://doi.org/10.1016/j.ijpharm.2019.118642.

70. O'Brien, J.B.; Wilkinson, J.C.; Roman, D.L. Regulator of G-protein signaling (RGS) proteins as drug targets: Progress and future potentials. J Biol Chem 2019, 294, 18571-18585, https://doi.org/10.1074/jbc.rev119.007060.

71. Dilly, S.; Lamy, C.; Marrion, N.V.; Liégeois, J.F.; Seutin, V. Ion-channel modulators: more diversity than previously thought. Chembiochem 2011, 12, 1808-1812, https://doi.org/10.1002/cbic.201100236.

72. Wu, P.; Nielsen, T.E.; Clausen, M.H. FDA-approved small-molecule kinase inhibitors. Trends Pharmacol Sci 2015, 36, 422-439, https://doi.org/10.1016/j.tips.2015.04.005.

73. Fischer, A.; Smieško, M. Ligand pathways in nuclear receptors. J Chem Inf Model 2019, 59, 3100-3109, https://doi.org/10.1021/acs.jcim.9b00360.

74. López-Otín, C.; Bond, J.S. Proteases: multifunctional enzymes in life and disease. J Biol Chem 2008, 283, 30433-30437, https://doi.org/10.1074/jbc.r800035200.

75. Buker, S.M.; Boriack-Sjodin, P.A.; Copeland, R.A. Enzyme-inhibitor interactions and a simple, rapid method for determining inhibition modality. SLAS Discov 2019, 24, 515-522, https://doi.org/10.1177/2472555219829898.

76. Shah, A.P.; Parmar, G.R.; Sailor, G.U.; Seth, A.K. Antimalarial phytochemicals identification from Euphorbia Hirta against plasmepsin protease: an In Silico Approach. Folia Med 2019, 61, 584-593, https://doi.org/10.3897/folmed.61.e47965. 